\title{
Ministros y precandidatos presidenciales en Chile 1990-2014
}

Ministers and pre-presidential candidates in Chile 1990-2014

\author{
Alejandro Olivares L. ${ }^{1}$ \\ FLACSO, Ecuador
}

\begin{abstract}
RESUMEN ¿Pasar por un gabinete presidencial entrega alguna ventaja para ser considerado como precandidato presidencial? En Chile los gabinetes han permitido la figuración de líderes que podrían tener aspiraciones presidenciales. Distintos factores podrían influenciar esta posibilidad tales como ser la cara visible del gobierno, las redes o que los votantes son influenciados por los medios de comunicación y las redes sociales debido a su poderoso rol en la sociedad, por lo tanto, ellos son esenciales para configurar nuevos liderazgos políticos. A pesar de dicha realidad, la academia no ha realizado la suficiente investigación sobre la vinculación entre ser ministro y ser precandidato presidencial. En este contexto, el artículo examina las posibles dimensiones de dicha vinculación.
\end{abstract}

PALABRAS CLAVE Gabinetes, precandidatos presidenciales, Chile.

ABSTRACT Is being a minister an advantage to those seeking nomination as a presidential candidate? In Chile, leaders who might have presidential aspirations often emerge from cabinets. Several factors could influence this route to candidacy, such as being the visible face of the government, participation in networks, or the fact that voting is influenced by media and social media due to their powerful role in the society, making them an essential element in the formation of new political leaders. Despite this situation, researchers and experts have not investigated sufficiently the link between being a minister and 
being a pre-presidential candidate. The present article examines the possible dimensions of this link.

KEYWORDS Cabinets, pre-presidential candidate, Chile.

\section{Introducción ${ }^{2}$}

Cuando en enero de 2018, el presidente electo de Chile (Sebastián Piñera) dio a conocer el gabinete que lo acompañarían en su segunda presidencia, el sistema político se sorprendió al confirmar que Alfredo Moreno, excanciller del primer gobierno de Sebastián Piñera (2010-2014) y expresidente de la Confederación de la Producción y del Comercio (2017-2018), asumiría como titular de Desarrollo Social. La sorpresa fue mayor cuando se indicó que este ministerio pasaría a ser parte del comité político del gobierno $^{3}$. La especulación de la prensa fue que Alfredo Moreno tenía intenciones de ser candidato presidencial ${ }^{4}$.

Este ejemplo ilustra lo que es un precandidato. En la práctica política la figura del precandidato es clara, se trata de una figura política no institucionalizada, pero formalizada por los actores. Se entiende, al menos, de dos maneras. Desde una visión, se trata de personas que están en campaña antes de que el periodo electoral se inicie, antes de que se abra la posibilidad de inscribir las candidaturas. Desde otro punto de vista, se trata de personas que, teniendo popularidad, son reconocidos por otros como potenciales candidatos. Esto último suele pasar con los ministros por la naturaleza de su cargo, cuando adquieren popularidad y se acercan las elecciones, suelen ser considerados como potenciales candidatos. Los miembros del gabinete, en particular, y ninguna autoridad política de confianza, en general, pueden reconocer abiertamente sus aspiraciones presidenciales. El reconocerlas significaría tener que renunciar al cargo, ya que pueden ser acusados de utilizar su posición para obtener ventajas. Solo se sabe que son candidatos al momento que abandonan sus cargos para iniciar

2. El autor agradece los comentarios y la ayuda de Camila Carrasco-Hidalgo, Bastián GonzálezBustamante y Gustavo Pérez A.

3. El comité político es una institución informal de asesoría directa del Presidente. Está conformado por los titulares del Ministerio del Interior; Ministerio Secretaría General de la Presidencia (SEGPRES); Ministerio Secretaría General de Gobierno (SEGEGOB); Ministerio de Hacienda. Tal como señalan EGAÑA y CHATEAu (2011), p. 142: "Este comité, por lo general, se reúne semanalmente a tratar los temas más significativos de la contingencia en la marcha del gobierno. Este Comité no tiene una existencia formalizada por medio de un decreto presidencial, sino que se ha creado en virtud de decisiones presidenciales en cada período".

4. Véase "La apuesta 'social' de Alfredo Moreno en el nuevo gobierno de Piñera y el vacío que deja en la CPC". Disponible en www.elmostrador.cl/mercados/2018/01/23/la-apuesta- politica-de-alfredo-moreno-de-repetirse-el-plato-con-pinera-y-el-vacio-que-deja-en-la-cpc/ [15 de septiembre de 2018]. 
una campaña política. En la literatura no existe una definición del precandidato presidencial. Cuando se analizan revistas especializadas en ciencia política, el concepto precandidato va asociado al análisis coyuntural (es común encontrar el concepto en anuarios y en artículos sobre casos), sin embargo, es un concepto sobre el que no se teoriza. Es decir, no existe una conceptualización sobre cuáles son las condiciones necesarias y suficientes para ser considerado como tal, a su vez, tampoco existe análisis de las variables comunes de las personas que son consideradas precandidatas.

La supuesta vinculación entre integrar un gabinete y una posible candidatura presidencial estaría explicada por las dinámicas de los sistemas presidenciales. En este tipo de gobierno, los ministros pueden asumir tareas de gestión política, dirección técnica y liderazgo mediático con cierto grado de independencia del jefe de gobierno. Esta independencia puede entregar beneficios a ambas partes, por ejemplo: ante una buena gestión puede existir popularidad para el Presidente y para el gabinete. Del mismo modo, cuando se implementan políticas públicas de ajuste que resulten problemáticas, el gabinete pueden ser el primer frente para las críticas de los ciudadanos disconformes, con dicho paquete de medidas, evitando, así, que estas lleguen al Presidentes.

Si todo sale bien para el gobierno y, aun así, el Presidente no puede ir a la reelección, parece lógico que las figuras que lo reemplacen y que, por tanto, aseguren continuidad de la gestión, estén en el gabinete. No obstante, la presencia de potenciales candidatos en los gabinetes ha sido un tema sobre el cual la investigación académica no ha prestado atención. La literatura especializada en selección de candidatos se centra en el papel de los partidos ${ }^{6}$. Esto es entendible, ya que muchas veces no son candidatos, sino que solo llegan a ser potenciales, esto es, solo alcanzan a precandidatos. En este sentido, lo lógico sería que la incipiente literatura sobre gabinetes se hiciera cargo de esta temática, en razón de poder determinar el impacto en la carrera de los sujetos al paso por los gabinetes. El proceso de nombramiento de ministros puede ser entendido desde dos ángulos ${ }^{7}$. El primero, entiende a la formación de gabinetes como el resultado de una serie de pugnas, negociaciones, compensaciones y equilibrios de poderes, que se anteponen al proceso de toma de decisiones del Presidente. La segunda perspectiva, asume que la formación del gabinete es de exclusiva responsabilidad del jefe del Ejecutivo, quien, por encima de cualquier discusión, con su base electoral (partido o coalición), va a realizar los nombramientos, pudiendo incluir a actores que no están en el campo político. En cualquiera de las dos dimen-

\footnotetext{
5. ALTMAN y CASTIGLIONI (2009).

6. HAZAN y RAHAT (2010); SIAVELIS y MORGENSTERN (2009).

7. RODRÍGUEZ (2009).
} 
siones, el estudio de la formación de gabinetes permite identificar dinámicas internas de cada sistema político ${ }^{8}$.

Considerando la ausencia de teorías sobre los ministros que son precandidatos presidenciales, se evalúan desde una lógica inductiva, los factores que podrían explicar por qué algunos integrantes del gabinete pueden ser considerados como potenciales candidatos presidenciales. A partir del examen de un caso en particular (Chile) se identifican algunas características de los ministros que eventualmente los hacen más propensos a ser precandidatos. El artículo está estructurado de la siguiente manera: en la primera parte se describen las variables del análisis. En la segunda parte se analiza la confirmación de gabinetes y el proceso de elección de los candidatos de las coaliciones oficialistas. La descripción va acompañada del análisis de la literatura. Luego, se analizan los factores que determinan la posibilidad de ser candidato presidencial siendo ministro, para ello se realiza un análisis de regresión con el modelo logit. El último apartado corresponde a la conclusión.

\section{Método}

Como se ha señalado, el artículo analiza los factores que podrían explicar por qué algunos ministros pueden ser considerados como precandidatos presidenciales. A falta de una teoría particular, se examinan algunos elementos de la teoría de los gabinetes y de la teoría de las elites. Del mismo modo, a modo de controlar los efectos, se agregan algunos elementos del entorno.

Asumiendo lógicas de los gabinetes, se incluye en el análisis si el ministro milita o no si en el partido del Presidente. Con ello se busca determinar en qué medida este vínculo se podría transformar en una herramienta estratégica para incluir en el gabinete a un potencial sucesor. Esta idea se genera a partir de una suerte de "bono presidencial" a la imagen del "bono formador" en los sistemas parlamentarios. El Presidente podría beneficiar a su partido en dos sentidos: por una parte, mayor número de ministerios y, por otra, con ministerios más visibles. En el sistema presidencial, la designación del gabinete y la salida de los ministros son decisiones que recaen en el Presidente, quien siempre actúa como el formateu ${ }^{9}$. El Presidente es el único actor con potencial para vetar cualquier alianza alternativa opuesta a sus intereses ${ }^{10}$. Existen dos teorías que podrían explicar los criterios utilizados por los presidentes para distribuir ministerios entre sus $\operatorname{socios}^{11}$. La primera teoría señala que los ministerios son repartidos de manera proporcional según el tamaño de cada partido en el Poder Legislativo. La segunda sostiene que en la división de ministerios el Presidente hace

8. DOGAN (1979).

9. ALEMÁN y TSEBELIS (2012).

10. ALTMAN (2000), p. 235.

11. Op. cit., p. 263. 
prevalecer un equilibrio entre todos los partidos, pero se reserva una amplia cuota del Ejecutivo para distribuir cargos entre los militantes de su facción o partido. Dependiendo del tamaño (fuerza electoral) del partido del Presidente, esa porción puede llegar a ser $50 \%$ del gabinete ${ }^{12}$.

Desde la teoría de los gabinetes también se incluye la duración en el cargo. Se asume que en la medida de no desarrollarse ajustes al gabinete existe compromiso con los partidos y su coalición ${ }^{13}$. Complementando lo anterior, se plantea que la rotación y los cambios de gabinete serían herramientas de ajuste que permiten hacer frente a shocks externos impredecibles ${ }^{14}$. Se asume, por lo tanto, que los ministros que sobreviven a los cambios son aquellos que después de la crisis (shocks) salen fortalecidos. Por ello, la duración en el cargo se utiliza como un indicador de méritos políticos codiciados para ser un potencial candidato. También se asumen que no es lo mismo estar mucho tiempo en un ministerio político, social, económico o mixto. Los ministerios sociales y políticos permiten un contacto más directo con la ciudadanía, mientras que los ministerios económicos tienen mayor visibilidad política. Los ministerios mixtos, por lo general, suelen conllevar tareas que mezclan lo político, lo económico y lo social.

A partir de las características personales de los sujetos (capitales) ${ }^{15}$, se construyó una tipología de los perfiles. Combinando capitales técnicos (credenciales académicas) y políticos (militancia, activismo político y cargos políticos desde el retorno a la democracia). Se consideró como suficiente credencial académica que los agentes tuviesen una profesión tecnocrática (ingenieros, economistas). Como evidencia de los recursos políticos, se consideró que los ministros fuesen militantes de partidos o que hubiesen desarrollado actividades partidistas (por ejemplo, ser director de un centro de estudios de un partido). Para la tipología se consideran solo la trayectoria a partir de 1990. La interacción de ambas variables permite la identificación de cuatro perfiles: tecnócrata, technopols, político y outsider, tal como se señala en la tabla 1.

Tabla 1. Tipología de ministros

\begin{tabular}{|l|l|l|c|}
\hline \multirow{4}{*}{ Creencias políticas } & Tiene & Políticos & Technopols \\
\cline { 2 - 4 } & No tiene & Outsider & Tecnócrata \\
\cline { 2 - 4 } & & No tiene & Tiene \\
\cline { 2 - 4 } & & \multicolumn{2}{|c|}{ Capital técnico } \\
\hline
\end{tabular}

12. Op. cit., p. 235.

13. MARTÍNEZ-GALLARDO (2012a).

14. MARTÍNEZ-GALLARDO (2012b).

15. GONZÁLEZ-BUSTAMANTE (2014), p. 61. 
Esta tipología tiene un arraigo en la literatura. Los ministros que solo poseen militancia son considerados como "políticos", ya que es posible que su nombramiento se deba a su condición de militante. Por último, los ministros que no tenían credenciales políticas ni capital técnico, fueron considerados como outsiders. Por su parte, los technopols corresponden a agentes que tienen una trayectoria académica y que, además, poseen influencia en la política de su país. Son un particular tipo de personas en las cuales recaen, al mismo tiempo, dos tipos de capital: los recursos técnicos y los recursos políticos, que se expresan por la ostentación de posiciones individuales o colectivas antes de alcanzar su primer nombramiento ${ }^{16}$. Los tecnócratas corresponden a personas que tienen justificada su nombramiento por sus credenciales académicas. Se consideró como tales a quienes tenían, antes de asumir el cargo, experiencia previa en el Estado en cargos de responsabilidad administrativa ${ }^{17}$ (por ejemplo, jefe de unidad o de gabinete, coordinador, profesional de alto nivel). Estos cargos se obtuvieron a pesar de que el sujeto carecía de militancia política, no tenían experiencia en el Congreso ni habían sido líderes de partidos. Se asume que su trayectoria y experiencia fue consecuencia de contar con una "profesión tecnocrática".

De manera alternativa a la tipología se considera la posibilidad de que el capital familiar o la trayectoria pública antes del golpe de Estado tengan influencia en la posibilidad de ser precandidato. Considerando que en la tipología solo se integran datos desde 1990, en se incluye en el modelo una variable con la trayectoria pública de los sujetos antes de 1973. Otra variable que se incluye como alternativa al perfil, es el capital familiar, es decir, contar con parentescos directos en política (padres, tíos, cónyuges y hermanos). El capital familiar se incluye en análisis estadísticos para ver si tienen una expresión más allá de la teorización ${ }^{18}$.

También como elemento de contexto se incluye la popularidad de los ministros. Se asume que son populares cuando son incluidos en la encuesta del Centro de Estudios Públicos ${ }^{19}$. Teóricamente los actores políticos que son populares siempre se transforman en potenciales candaditos. En contra partida, los políticos con presencia nacional que no son populares tienen pocas posibilidades de optar a cargos de elección como la presidencia.

16. JOIGNANT (2011).

17. SILVA (2010).

18. GONZÁLEZ-BUSTAMANTE (2014); JOIGNANT (2014).

19. Con algunas variaciones en el tiempo, la pregunta del CEP dice: "Las siguientes personas tienen o han tenido diversos grados de notoriedad en la vida pública nacional. Indique, usando los términos de esta tarjeta, ¿cuál término describe mejor su opinión sobre cada persona?”. Los resultados que presenta la encuesta son: "Opinión Positiva (quienes conocen)". 
En el modelo se incluyen elementos que pueden ser relevantes dado el contexto, por ejemplo el estado de la economía. Para ello se incluyen dos variables: Producto Interno Bruto (PIB) y Índice de Precios al Consumidor (IPC). El PIB expresa el crecimiento de la economía y el IPC la inflación. Es posible asumir que en la medida que la economía va creciendo y que la inflación no sube, la economía es fuerte. En este contexto es posible asumir que los ministros oficialistas tendrán incentivos para ser candidatos. Con el fin de no sobreestimar el modelo, se incluye en análisis la variación de ambos $\left(t_{1}-t_{2}\right)$. Para tal efecto, se considera como $t_{1}$ el nombramiento en el cargo cómo $t_{2}$ la fecha de salida.

Por último, se incluye en el análisis una variable sobre la orientación ideológica de los partidos políticos. Para asignar un lugar en la escala izquierda derecha se utilizó la encuesta del Observatorio de Elites Parlamentarias de América Latina. En la encuesta se les solita a los diputados en ejercicio entre 1990 y 2010 calificar a los partidos en una escala que oscila entre izquierda (1-2) y derecha (9-10). Para nuestro análisis, considerando que la variación en la respuesta a esta pregunta es mínima, se utilizan los promedios del periodo. Con esto los partidos se ordenan de la siguiente manera: PS 2,43; PRSD 3,66; PPD 3,845; PDC 4,76; RN 7,66; UDI 9.46. Para los independientes se consideró el valor como perdido, al menos que tengan cercanías a expresas con un partido.

La variable dependiente es "que un ministro sea mencionado como precandidato presidencial" ( 1 = sí; o = no). Como se ha señalado en la literatura especializada, no existe definición de precandidato. Por ello es que considera como 1 a los ministros que en la prensa fueron señalados como tal, aun si el aludido no lo hubiese señalado en público. Para codificar la variable se analizó dos diarios de circulación nacional (El Mercurio y La Tercera). Es importante aclarar que no se consideró en análisis las personas que fueron precandidatos después de dejar el gabinete. Por ejemplo, no se incluye a Jorge Arrate (ministro con Patricio Aylwin y precandidato en 2009), a Ximena Rincón (ministra con Michelle Bachelet y precandidata en 2013), Felipe Kast (ministro con Sebastián Piñera y precandidato en 2017).

La investigación busca probar si la posibilidad de ser precandidato estando en el gabinete aumenta en la medida que tiene un perfil político o en la medida que se está mucho tiempo en el cargo. De manera alternativa, como segunda hipótesis, se asume que, con independencia de la orientación ideológica, la popularidad puede ser la variable que explique que un ministro sea considerado como potencial candidato a presidente. Para comprobar esta hipótesis y considerando las características de la variable dependiente, se utiliza una de regresión logística (logit) con efectos marginales condicionales. En el modelo se verificó que no existiese multicolinealidad mediante el análisis de correlaciones entre todas las variables. Tal como se demuestra en la matriz en anexo no existen correlaciones fuertes (superiores a 0.7) por lo que se descarta la 
posibilidad de multicolinealidad (véase anexo). Para controlar el efecto del tiempo, el análisis fue realizado considerando a cada uno de los gobiernos como conglomerados. Es decir, el modelo es robusto con errores estándares ajustados para cinco clusters (uno por cada gobierno). Para las variables que se generaron a partir del perfil de los ministros, la categoría de referencia es outsiders. En el caso del tipo de ministerio la categoría de referencia son los ministerios mixtos. Para las variables de orientación ideológica la categoría de referencia es la izquierda.

Tabla 2. variables

\begin{tabular}{|l|l|}
\hline Variable & Definición \\
\hline Sexo & $0=$ mujer;1=hombre \\
\hline Edad & Edad al asumir el cargo. \\
\hline Trayectoria pre 1973 & $0=$ no; $1=$ sí \\
\hline Milita en partido del Presidente & $0=$ no; $1=$ sí \\
\hline Capital familiar & $0=$ no; $1=$ sí \\
\hline Duración en el cargo & Días en el cargo. \\
\hline Variación PIB & $\begin{array}{l}\text { Variación (resta) del valor del año que asume y del año que } \\
\text { sale. }\end{array}$ \\
\hline Variación IPC & Variación del valor del mes que asume y de año que sale. \\
\hline Orientación política & $\begin{array}{l}\text { PS 2,43; PRSD 3,66; PPD 3,845; PDC 4,76; RN 7,66; UDI } \\
9,46 .\end{array}$ \\
\hline Perfil (Tipología de ministros) & Dummy para cada una de las categorías. \\
\hline Tipo de Ministerio & $\begin{array}{l}\text { Según los fines principales de la cartera, se consideró cua- } \\
\text { tro tipos de ministerios: Políticos (Interior, SEGEGOB, } \\
\text { SEGPRES, Relaciones Exteriores, Defensa); Económicos } \\
\text { (Hacienda, Económica, Medio Ambiente, Obras Públicas, } \\
\text { Trasportes, CORFO); Social (Justicia, Salud, Mujeres, Plani- } \\
\text { ficación, Cultura, Educación, Vivienda), Mixto (Agricultura, } \\
\text { Bienes Nacionales, Trabajo). Dummy para cada una de las } \\
\text { categorías. }\end{array}$ \\
\hline Nombrado en encuesta CEP & \begin{tabular}{l} 
0=no; 1=sí. \\
\hline
\end{tabular} \\
\hline
\end{tabular}

\section{Precandidatos y candidatos en los gabinetes}

A lo largo de la historia de Chile, los gabinetes han sido una instancia que ha permitido la figuración de líderes políticos, llegando varios de ellos a ser candidatos presidenciales después de su paso por algún ministerio. Algunos llegaron a ser presidentes en el periodo posterior al cual estuvieron en el gabinete (fueron ministros del gobierno que los antecedió como presidentes: Juan Antonio Ríos, Ricardo Lagos y Michelle Bachelet. Fueron ministros en algún momento de su carrera política antes de ser presidente: Pedro Aguirre Cerda, Carlos Ibáñez, Jorge Alessandri, Eduardo Frei Montalva, Salvador Allende). 
Es una práctica común en la política chilena que la primera exploración de candidatos presidenciales del oficialismo se haga en el gabinete. Teniendo esto como antecedente, a continuación se analizan los procesos de selección de candidatos presidenciales en el oficialismo, dando énfasis en el proceso de definición de las candidaturas cuando en ellas participan ministros. En la primera parte de la sección se analizan los gobiernos de centro izquierda (1990-2010) y luego el gobierno de derecha (20102014). Para ver trabajos que podrían servir como marco general para estudiar este y otros temas de los gabinetes antes del golpe de Estado ${ }^{20}$.

\subsection{Los gobiernos de la Concertactación}

El periodo 1990-2010 se caracteriza, desde el punto de vista político, por la continuidad de los gobiernos. La Concertación, coalición de centro-izquierda, logró cuatro presidencias consecutivas, algo inédito en la historia política del país. Esta coalición fue creada a fines de la década de 1980 ochenta bajo el nombre de Concertación de Partidos por el No, con el propósito de lograr el retorno a la democracia, utilizando los mecanismos institucionales ${ }^{21}$. Esta alianza estaba formada por más de diez partidos y movimientos que optaron por la vía institucional y no armada para derrotar a Augusto Pinochet. Esta coalición, logró articular partidos y movimientos de centro e izquierda $^{22}$. Al inicio fueron más de veinte partidos y movimientos que formaron la coalición. Su número fue reduciéndose por diversos motivos (entre ellas fusiones de partidos, desapariciones de grupos). El proceso de institucionalización de la Concertación termina en 1994 con la fusión entre el PR y el PSD, desde ese momento la coalición está conformada por cuatro partidos: PDC, PS, PPD y PRSD (el PH abandonó el pacto a fines del gobierno de Patricio Aylwin) ${ }^{23}$.

20. OLIVARES (2017), ARELLANO, TORO y NOVOA (2018) y SIAVELIS y GALVAN (2015).

21. GARRIDO y NAVIA (2005), p. 174.

22. GARRIDO y NAVIA (2005).

23. Para la creación de la Concertación es posible identificar dos objetivos; primero poner fin al régimen burocrático autoritario y, en segundo lugar, se propuso ser una coalición estable que se transformara en una alternativa de conducción política para la transición a la democracia. El primero se logró en octubre de 1988 con el triunfo en el plebiscito. El segundo fue lográndose en diversas etapas, destacando tres hitos importantes: las negociaciones con los militares para las reformas constitucionales de 1989, la elección de un candidato único para la elección presidencial del mismo año y el triunfo en las elecciones de ese año. La concreción de ambos objetivos, más la preocupación por los derechos humanos, pueden ser considerados cómo la coyuntura crítica que explica los procesos de toma de decisiones en los años siguientes. AVENDAÑO (2010). 
En términos políticos sus gobiernos fueron estables. Existan mecanismos de coordinación interna para resolver conflictos ${ }^{24}$. Gran parte de la estabilidad de esta coalición se debe a la conducción política del primer gobierno. Existe amplio consenso para calificar al gobierno de Patricio Aylwin (1990-1994) como estable y de generador de consensos, tanto dentro de la Concertación como entre los distintos sectores políticos del país ${ }^{25}$. Esto en gran parte porque al interior de la coalición uno de los puntos con más consenso fue la idea de no repetir la ingobernabilidad de la Unidad Popular. "El temor de repetir la experiencia de la ingobernabilidad de la Unidad Popular (UP) llevó a los líderes de la Concertación al desarrollo las reglas informales para disminuir los posibles conflictos en la asignación de posiciones políticas" ${ }^{26}$.

La estabilidad lograda por el primer gobierno de la Concertación puede ser explicada por la existencia de reglas informales para la designación del gabinete. Según el autor se desarrollaron dos reglas ${ }^{27}$. La "integración horizontal", cada ministro tenía como subsecretario a un "colega perteneciente a otro partido de la Concertación" ${ }^{28}$ y que los ministerios más importantes (los políticos) eran ocupados por militantes del partido del Presidente ${ }^{29}$. La interacción entre ambas reglas generó mecanismos que facilitaron la confianza entre los partidos y entre los militantes de la coalición. Este fenómeno se ha definido como suprapartidismo. El suprapartidismo da al jefe de gobierno autonomía real para decidir sobre su gabinete ${ }^{30}$. Los líderes de los partidos aceptaron que el Presidente tenía la libertad para estructurar su gabinete, pero, al mismo tiempo, el Presidente fue el responsable de equilibrar la presencia del partido en el gabinete generando que "los miembros del gabinete eran leales al Presidente primero y luego a su partido" ${ }^{31}$. La regla de los cuatro gobiernos de la Concertación para mantener la unidad de la coalición fue la proporcionalidad, "la que algunas veces, se acercó a perfectas proporciones matemáticas" ${ }^{32}$.

24. El paso de los años y desgaste por el ejercicio del poder significaron problemas internos, véase DÁVILA, OLIVARES y AVENDAÑO (2013).

25. SIAVELIS (1999), FUENTES (1999), GONZÁLEZ-BUSTAMANTE y OLIVARES (2016).

26. DÁVILA (2011).

27. REHREN (1993).

28. Op. cit., p. 71.

29. REHREN (1993), p. 72.

30. DÁVILA (2011).

31. Op. cit., p. 78.

32. ALTMAN (2008), p. 269. 
Como consecuencia de la autonomía presidencial para nombrar a sus ministros, a partir del gobierno del presidente Eduardo Frei Ruiz-Tagle es posible identificar una suerte de "bono presidencial" (a la imagen del "bono formador" en sistemas parlamentarios) que beneficia al partido del Presidente en dos sentidos: mayor número de ministerios, con ministerios más. En los periodos que gobernó la Concertación, se asumió que el partido formador era el partido del Presidente, lo que le otorga una pequeña ventaja sobre el resto de los miembros de la coalición ${ }^{33}$. Del mismo modo, existió un reforzamiento de las candidaturas presidenciales insiders. Los presidentes de la concertación tenían historia en los partidos institucionalizados, con gran respeto por los cuadros políticos, la vida militante dentro del partido y la disciplina interna ${ }^{34}$.

Lo anterior es evidente al finalizar el periodo del gobierno de Patricio Aylwin. Existieron en la coalición varios líderes populares con intención y posibilidad real de ganar la elección presidencial. El proceso de nominación del candidato presidencial de la Concertación se inició, con la búsqueda informal de eventuales candidato ${ }^{35}$. Por el lado del PDC estaba Eduardo Frei Ruiz-Tagle, hijo del expresidente Eduardo Frei Montalva (1964-1970), senador en ejercicio y presidente del partido (en las elecciones internas de la DC en 1991 obtuvo cerca del 70\% de los votos). Desde la izquierda de la alianza el candidato era Ricardo Lagos, líder del socialismo, fundador del PPD, excandidato a senador y ministro de Educación de Patricio Aylwin. A pesar de la ventaja de ambos había otros líderes del PDC que también tenían aspiraciones presidenciales. Los senadores Andrés Zaldívar, Gabriel Valdés y el titular de Hacienda, Alejandro Foxley $^{36}$ fueron precandidatos ${ }^{37}$, pero el avasallador avance de Eduardo Frei Ruiz-Tagle los dejó sin oportunidades reales ${ }^{38}$.

Una vez definidos los candidatos (Ricardo Lagos y Eduardo Frei Ruiz-Tagle) la Concertación enfrentó el desafío de diseñar un mecanismo que permitiera que el nombramiento del candidato único por medio de un proceso que asegurara amplios niveles de legitimidad, tanto dentro de los partidos como en la ciudadanía ${ }^{39}$, por eso se diseñó un mecanismo que aseguraba la participación de los ciudadanos y de los partidos de la coalición: una convención con tres mil delegados que serían seleccio-

\section{DÁVILA (2011).}

34. ALTMAN (2008).

35. NAVIA (2005), pp. 441-442.

36. Como recuerda NAVIA (2005), p. 443. "Foxley señaló en El Mercurio que 'le hubiera gustado ser Presidente'. Aunque esta declaración no transforma al actor en precandidato oficial, sí indica que sus aspiraciones presidenciales existieron.

37. OTANO (1995), p. 187.

38. NAVIA (2005), p. 443.

39. NAVIA (2005). 
nados de dos maneras: por votación se elegirían a mil ochocientos, los mil doscientos restantes serian nombrados por los partidos ${ }^{40}$, "de esta forma, el PDC nombraría a un $54 \%$ de los delegados no electos, el PR nombraría a un 9,5\%, y el PS-PPD nombraría a un $33 \%$, el resto de los delegados no electos serían nombrados por la Social Democracia y otros grupos menores" ${ }^{41}$. El resultado de las elecciones confirmó la ventaja que tenía Eduardo Frei Ruiz-Tagle, como consecuencia del pacto entre el PDC y el PR. En total, el candidato de la DC logró reunir a 1924 representantes, mientras que Ricardo Lagos a $1076^{42}$. Luego, y sin mucha sorpresa, el candidato único de la Concertación obtuvo un 58\%, el candidato de la derecha, Arturo Alessandri solo un 24\%.

La segunda administración de la Concertación (1994-200o), liderada por Eduardo Frei Ruiz-Tagle, asumió un discurso marcado en la modernización del Estado y de la gestión pública, lo que ha llevado a algunos analistas a asumir que fue un gobierno en el cual existió una mayor preponderancia de los criterios técnicos por sobre los políticos "la administración Frei privilegió a los técnicos y tecnócratas, pues el énfasis de su administración estaba puesto en el desarrollo de la Modernización del Estado" ${ }^{43}$. La detención de Augusto Pinochet en Londres borró esta idea de un gobierno técnico, y les entregó a los políticos del gabinete un papel central en la agenda ${ }^{44}$. Dos tipos de políticos formaron parte de la élite ministerial del gobierno de Eduardo Frei Ruiz-Tagle; ${ }^{45}$ los dirigentes de partidos y los dirigentes de la DC. Esto explica que el supuesto bajo el cual se nombró su primer gabinete fue que al incluir como ministros a los líderes de los partidos se crearía una aproximación entre los intereses de los miembros de la coalición, además, se solucionarían los problemas de comunicación entre el gobierno y los partidos ${ }^{46}$.

Dicha lógica fue conocida, en los medios, como troika o "alianza de tres personas con idéntico poder y las mismas atribuciones", estos tres dirigentes de partidos, ahora ministros, eran Genaro Arriagada (DC) en la Secretaría General de la Presidencia, Víctor Manuel Rebolledo (PPD) en la Secretaría General de Gobierno y Germán Correa (PS) en el Ministerio del Interior ${ }^{47}$. El otro líder de partido en el gabinete Luis Maira (secretario general del PS) asumió en el Ministerio de Planificación, con este

40. AUTH (2005), p. 11.

41. NAVIA (2005), p. 444.

42. NAVARRETE (2005).

43. AVENDAÑO (2010b), p. 172.

44. DÁVILA (2011).

45. HIDALGO (2011), p. 123.

46. OTANO (2006), p. 240.

47. Op. cit., p. 241. 
nombramiento el PS debió reelegir a sus dos máximas autoridades. El hecho de que Eduardo Frei Ruiz-Tagle nombrase como ministros a los dirigentes de los partidos provocó la excesiva politización del gabinete lo que dificultó la toma de decisiones ${ }^{48}$. Por esta razón este diseño no podía durar mucho tiempo y estaba destinada al fracaso.

El excesivo énfasis en la modernización y no en la agenda política fue, en cierta medida, una fuente de desilusión para los militantes de la Concertación ${ }^{49}$. A esto se debe agregar que el estilo de conducción presidencial provocó tensiones en la coalición, por ejemplo con en el nombramiento y posterior salida de Germán Correa del Ministerio del Interior. En un primer momento el PS se opuso a que su presidente asumiera como ministro y manifestó su malestar. A los seis meses de gobierno el Presidente decide sacarlo del cargo. Lo hace por carta y sin consultar, ni informar al PS. En su reemplazó asumió Carlos Figueroa. Las tensiones en la coalición son evidentes y se inicia una serie de discusiones y debates sobre el presente y futuro de la coalición, que marcan un punto de inflexión dentro de la Concertación. El debate entre quienes están "complacidos" con las obras de la alianza y quienes se "flagelan" por todo lo que no se ha avanzado, se toma de la agenda interna. El escenario interno se complica aún más con las elecciones legislativas del año 1997 (donde la DC, principal partido del pacto, cae desde un $27,12 \%$ al $12,55 \%$ ) y con las consecuencias de la crisis financiera del año 1998.

En lo que respecta a la relación de los presidenciales en el gabinete, el gobierno de Eduardo Frei Ruiz-Tagle optó porque los posibles candidatos asumieran ministerios técnicos y no políticos, con el fin de no entorpecer la coordinación dentro de la alianza. Por ello es que a Alejandro Foxley se le ofreció el Ministerio de Educación y a Ricardo Lagos el de Obras Públicas ${ }^{50}$ Alejandro Foxley no aceptó el ministerio. Por su parte, Ricardo Lagos supo transformar un ministerio técnico en una plataforma presidencial. El otro precandidato que surgió entre los ministros fue Soledad Alvear. La ex Ministra de Patricio Aylwin y titular de Justicia del gobierno de Eduardo Frei Ruiz-Tagle emergió como una líder que ante la opinión pública tenía muchas posibilidades de ser candidata presidencial. Aunque su opción no prosperó, pues el cupo de la DC fue tomado por el senador Andrés Zaldívar, su popularidad fue un activo que se mantuvo en alza, a tal punto que se transformó -durante la segunda vuelta- en la jefa de campaña de Ricardo Lagos.

Al finalizar el gobierno, la Concertación realizó elecciones primarias abiertas y vinculantes para definir al candidato único de la alianza. Estas elecciones fueron vistas como una instancia de movilización interna que permitiría consolidar la unidad

48. GARRETÓN (2003), p. 55.

49. HIDALGO (2011), p. 124.

50. OTANO (2006), p. 422. 
de la coalición ${ }^{51}$. De hecho, la popularidad de Ricardo Lagos "se transformó en apoyo electoral en dichas primarias, lo cual sumado a una rápida aceptación de su derrota por parte del candidato PDC (el Senador Andrés Zaldívar) consolidaron la unidad de la alianza" ${ }^{2}$.

Considerando la popularidad que tenía Ricardo Lagos, producto de su paso por los gabinetes de Patricio Aylwin y Eduardo Frei Ruiz-Tagle, así como por su papel en la transición, la elección presidencial que lo enfrentó con el UDI Joaquín Lavín resultó contra todo muy difícil. Nadie, o muy pocas personas, esperaban que hubiese segunda vuelta. El resultado de la elección estuvo mediado por los problemas que la administración Frei tuvo para enfrentar la crisis económica internacional y por los temores que representaba la figura de Ricardo Lagos, por su orientación ideológica ${ }^{53}$.

Lo estrecho de la victoria de Ricardo Lagos, y los temores de algunos sectores de la sociedad por tener en el gobierno al líder de la izquierda de la Concertación (PPDPS) influyeron en el diseño de los gabinetes ${ }^{54}$. Lagos sacrificó el bono formador y le dio ventaja al Partido Demócrata Cristiano, que tuvo el $49 \%$ de los ministros versus un 39,7\% de los militantes del PPD y del PS. En su gobierno, nombró ministros con trayectoria política, capacidad técnica y alta presencia mediática, lo que significó que varios de ellos fuesen populares en las encuestas ${ }^{55}$. También permitió que muchos tuviesen aspiraciones presidenciales. Por ejemplo, fueron nombrados como precandidatos José Miguel Insulza (ex Ministro con Eduardo Frei Ruiz-Tagle), Jaime Ravinet (exalcalde de Santiago), Michel Bachelet (ministra de dos carteras, Salud y Defensa), Soledad Alvear (ex Ministra de Patricio Aylwin y Eduardo Frei Ruiz Tagle).

Uno de los objetivos de este diseño de gabinete con sobre representación de la DC y con líderes políticos reconocidos, fue mitigar los temores que despertaba entre algunos sectores de la sociedad la llegada de un socialista al poder. El temor inicial dio paso, rápidamente, a una administración que gozó con altos y crecientes niveles de popularidad ${ }^{56}$. Aunque en algún momento hubo varios precandidatos hacia el final del gobierno de Ricardo Lagos la carrera al interior de la Concertación se concentró entre Michelle Bachelet (PS) y Soledad Alvear (DC). Una vez seleccionadas las dos candidatas presidenciales se definió la realización de una primaria abierta en julio de 2005. No obstante, dicho acuerdo, la candidata PDC abandonó la competencia en

51. OLIVARES, BAEZA, DÁVILA (2015).

52. NAVIA y JOIGNANT (2000).

53. NAVIA y JOIGNANT, 2000; FUNK (2006).

54. OLIVARES, BAEZA, DÁVILA (2015).

55. HIDALGO (2011) p. 148.

56. FUNK (2006). 
mayo de 2005, ya que las encuestas señalaban que no tenía ninguna posibilidad de vencer a la candidata del PS, quien se transformó en la primera mujer en llegar a la presidencia.

Asume la presidencia con un discurso centrado en los ciudadanos. Se produce un desplazamiento de los círculos más tradicionales de la Concertación como respuesta a la desafección ciudadana ${ }^{57}$. Al respecto, Varas señala que el primer gabinete de Michelle Bachelet se hizo "sin consulta con los partidos que la apoyaban" ${ }^{8}$. Esto más el estilo ciudadano que comprometió la Presidenta "distanciaron la gestión gubernamental de la parlamentaria y no ayudó a contener la indisciplina entre los congresistas, resultando en un Ejecutivo menos involucrado en la posterior crisis de la coalición de gobierno" ${ }^{59}$. Esto fue así hasta la crisis sub prime. El manejo de la crisis económica significó un aumento progresivo de la popularidad de la Presidenta y una aceptación por parte de los partidos y sus militantes del estilo de Michelle Bachelet.

En términos generales evaluar el recambio de líderes en los gabinetes de Michelle Bachelet es difícil, ya que depende de las variables que se consideren al hacer la evaluación. Si el recambio es entendido como que los exministros no participen en el gobierno, es factible decir que esto se logró, ya que la tasa de participación de estos en los gabinetes de la Presidenta es baja, solo un 17,4\% de los nombrados tenían experiencia previa en gabinetes, seis habían integrado en los gobiernos anteriores algún gabinete: Francisco Vidal (dos veces con Ricardo Lagos); Edmundo Pérez (dos veces con Ricardo Lagos); Sergio Bitar (durante el gobierno de Ricardo Lagos); René Cortázar (durante el gobierno de Patricio Aylwin), Yasna Provoste (durante el gobierno de Ricardo Lagos), Alejandro Foxley (durante el gobierno de Patricio Aylwin). No obstante, cuando analizan las trayectorias y se incluyen los cargos anteriores de los ministros (cargos de confianza política entre 1990 y 2005), se observa que los de Michelle Bachelet tuvieron un amplio recorrido en el Estado, un 74\% de ellos desempeñó dos o más cargos antes de ser nombrado.

Los gabinetes de Michelle Bachelet fueron de dos tipos, en la primera parte de sus gestiones se privilegió gente con perfil más técnico y durante la segunda se dio paso a un perfil más político. Esto no quiere decir que los técnicos fueran outsiders - un 74\% de ellos desempeñó dos o más cargos políticos o técnicos en los gobiernos anteriores-. Los gabinetes fueron conformados por militantes de partidos, solo cuatro ministros (que es equivale al $9 \%$ del total de ministros) fue no militante. En general se mantiene la lógica de reclutamiento insider, el 34\% de los ministros fueron del Partido Demócrata Cristiano, 28\% del Partido por la Democracia, 21\% del PS y $7 \%$ del Partido Radical Socialdemócrata.

57. LUNA y SELIGSON (2007).

58. VARAS (2010), p. 302.

59. Op. cit., p. 303. 
A diferencia de los presidentes que la antecedieron, no tuvo una estrategia de sucesión $^{60}$, por ello es que nadie en el gabinete era señalados entre los potenciales candidatos a la presidencia. El único que alguna vez fue mencionado como potencial candidato fue el ministro de Hacienda, Andrés Velasco. Con todo, los principales nombres eran el exministro José Miguel Insulza, la senadora Soledad Alvear y los expresidentes Ricardo Lagos y Eduardo Frei Ruiz-Tagle ${ }^{61}$, la falta de figuras con interés de ser presidenciales es consecuencia del perfil técnico de los gabinetes, lo que anuló las posibilidades de proyección de algún ministro ${ }^{62}$. Esta explicación puede ser cuestionada, ya que el presente análisis sugiere que los ministros del gobierno de la Presidenta eran personas con amplias redes en la Concertación y militantes de los partidos que integran dicho pacto. Pot último, y luego de varios problemas, el candidato presidencial de la Concertación fue el expresidente Eduardo Frei Ruiz-Tagle, que venció en la elección primaria a José Antonio Gómez, quien fue ministro de Justica durante el gobierno del propio Eduardo Frei. La elección primaria de la Concertación tuvo una consecuencia inesperada, el diputado socialista Marco Enríquez-Ominami, que tenía la intención de ser candidato presidencial. No participó del proceso, pues su partido no lo apoyó. Compitió como independiente con el apoyo de dos partidos. En el proceso varios militantes y simpatizantes de la Concertación dejaron la coalición para trabajar con él.

En la elección participaron el expresidente Eduardo Frei Ruiz-Tagle (Concertación), Sebastián Piñera (Coalición por el Cambio), Jorge Arrate (Juntos Podemos) y Marco Enríquez (Partido Humanista y Partido Ecologista). Pasaron a segunda vuelta los candidatos de los dos pactos tradicionales (Eduardo Frei y Sebastián Piñera). En la segunda vuelta se impuso el candidato de la derecha Sebastián Piñera, que con su triunfo logró que la derecha volviese al poder de manera democrática después de 58 años. Muchos de los dirigentes de la derecha chilena estuvieron en el poder durante la dictadura de Augusto Pinochet. Fueron ministros, subsecretarios, asesores y otros cargos de primera línea ${ }^{63}$.

\subsection{Los gabinetes de la derecha}

En este contexto de malestar con la Concertación, creciente movilización social y la elección presidencial de 2009 fue el momento de recambio político. Las elecciones fueron ganadas por el representante de la derecha Sebastián Piñera (2010-2014).

60. MORALES y NAVIA (2010), p. 27.

61. Op. cit., p. 31.

62. AVENDAÑO (2010), p. 12.

63. RUBIO (2014). 
Apoyado por la Coalición por el Cambio, alianza política que agrupó a dos partidos: Unión Demócrata Independiente (UDI) y Renovación Nacional (RN). Este bloque, que ha tenido diversos nombres (Democracia y Progreso, Participación y Progreso, Unión por el Progreso de Chile, Unión por Chile, Alianza por Chile), a pesar de tener dos partidos, tiene diversidad ideológica. Por una parte, la UDI es un partido de corte conservador en lo valórico y neoliberal en lo económico. RN tiene una orientación un poco más liberal en lo valórico y en economía es claramente neoliberal. Ambos partidos tienen una importante tradición pinochetista, aunque la UDI suele ser señalado como el partido que más defiende el legado de la dictadura.

El gobierno de Sebastián Piñera presenta lógicas muy similares a las desarrolladas por la Concertación, en el sentido de la libertad que tenía como gobernante para designar a sus ministros sin consultar con los partidos ${ }^{64}$. Su gabinete inicial contó con gente que tenía con poca experiencia política, pero con una amplia trayectoria en el campo empresarial y académico. Su gobierno debió enfrentar presiones como consecuencia del terremoto (por la reconstrucción de la zona afectada), movimientos sociales y por una serie de problemas contingentes, que, en gran medida, crecieron por los problemas de conducción política. Luego de casi dos años de gobierno acogió las presiones de los partidos oficialistas, quienes lograron un cambio de gabinete en el que los políticos con mayor experiencia se hicieron presentes, desplazando a los outsiders.

En su administración nombró a 52 ministros. De ellos doce son mujeres. La mayoría no militaba ( $52 \%)$. Cuando se analiza la totalidad de los nombramientos se observa que la mayoría de los ministros son militantes de partido sin experiencia en el Congreso (38,5\%), los que contaban con experiencia en el Legislativo fueron muy pocos (13,5\%). El segundo grupo en importancia en el gabinete fueron los outsiders al sistema político, que representaron el $28,8 \%$. En este grupo están personas que vienen del mundo empresarial, gerentes del retail, empresarios de sectores como salud y socios inversionistas de Sebastián Piñera. El porcentaje restante de los ministros presentaban credenciales técnicas (ingenieros con trayectoria académica, abogados vinculados a universidades privadas). Una de las críticas al gabinete es que se presentan muchos conflictos de interés. Por ejemplo, el titular de Salud era dueño de una las clínicas privadas más grandes del país ${ }^{65}$.

El gobierno de Sebastián Piñera debió enfrentar una serie de movilizaciones, entre ellas, de los estudiantes universitarios, las más grandes desde el retorno a la democracia $^{66}$. Esto provocó muchas tensiones dentro del gobierno, particularmente cuando el

64. GONZÁLEZ-BUSTAMANTE y OLIVARES (2018).

65. MAILLET, GONZÁLEZ-BUSTAMANTE y OLIVARES (2016).

66. TRICOT (2012); VON BÜLOW y BIDEGAIN (2015). 
tecnócrata, ministro de Educación Harald Beyer, fue acusado constitucionalmente y el senado instruyó su salida del cargo (2013). Fue acusado de no ejercer sus funciones de fiscalización contra el lucro en las universidades. La acusación promovida por la Concertación fue posible por el voto de congresistas que habían dejado de militar en los partidos de derecha y que ahora eran independientes.

El gobierno tuvo éxitos, que permitió a varios de sus ministros ser considerados como precandidatos presidenciales. Quizá el hito más importante en esta dirección fue el rescate con vida de los treinta y tres mineros tras el derrumbe de la mina San José en el norte de Chile. Este hito, le permitió al ministro de Minería Laurence Golborne transformarse en una figura popular, lo que rápidamente lo transformó en un precandidato presidencial. Además de él, fueron precandidatos los ministros Andrés Allamand (RN, titular de Defensa), Pablo Longueira (UDI, titular de Economía), Evelyn Matthei (UDI, titular del Ministerio del Trabajo). Todos, salvo Laurence Golborne, eran políticos con amplia trayectoria, ya que contaban con pasos por el Congreso (exdiputados y exsenadores) y por activa militancia en sus partidos.

La definición del candidato presidencial del oficialismo fue bastante complicada. La UDI definió como su candidato a Laurence Golborne. A muy pocos días de la inscripción de la candidatura para las elecciones primarias una serie de escándalos (la aparición de cuentas en paraísos fiscales y problemas en la empresa donde él fue gerente general antes de asumir en el gabinete) hicieron que declinase la candidatura. En esa coyuntura la UDI inscribió como candidato al exministro Pablo Longueira, quien venció en la primaria a Andrés Allamand. No obstante, a un mes de haber ganado la elección primaria renunció, argumentando problemas de salud. En su reemplazo asumió como candidata la exministra Evelyn Matthei. La elección presidencial estuvo marcada por el alto número de candidatos. Nueve personas compitieron a la presidencia, de ese número solo dos eran independientes (uno de ellos Tomás Jocelyn-Holt, quien obtuvo solo o,19\% de la votación, transformándose en el candidato presidencial menos votado de la historia de Chile). Los otros siete candidatos fueron apoyados por partidos y coaliciones de partidos. La elección se definió en segunda vuelta entre las candidatas de las coaliciones más grandes: Michelle Bachelet apoyada por la Nueva Mayoría (una coalición entre concertación, el Partido Comunista, Izquierda Ciudadana y MAS Poder) y Evelyn Matthei (Alianza por Chile, formada por RN y la UDI). Finalmente, Michelle Bachelet es electa como presidenta de Chile.

\section{Resultados}

La mayoría de los ministros son hombres. De los 232 ministros del periodo solo cincuenta fueron mujeres. Esto demuestra que aún es necesario avanzar en políticas de integración efectiva para las mujeres. A pesar de que al final del periodo examinado el número sube, esto no es una tendencia, ya que solo en el gobierno de Michelle Bache- 
let el número de ministras está cerca del cincuenta por ciento. Del mismo modo, la edad tiende a tener una distribución normal. Los grupos con menos población están en los extremos. Entre 31 y 40 años un 15,5\% y de 61 años en adelante un 12,5\%. El resto está en dos grupos entre 41 y 50 años (30,17\%) y entre 51 a 60 años $(41,81 \%)$.

Un 32\% de los ministros tienen trayectoria en Estado antes de 1973 (ministro, subsecretario, senador, diputado, alcalde, embajador político, gobernador, intendente y jefe de servicio o algún cargo de confianza política). La mayoría se concentran en los dos primeros gobiernos (Patricio Aylwin y Eduardo Frei Ruiz-Tagle). Respeto a los perfiles de los ministros se destaca que la mayoría son políticos $(74,6 \%)$. Los tecnócratas representan al 9,05\% y los technopols al 11,2\%. Por último, los outsiders representan al 5,17. Los militantes del partido del Presidente representan un 33\% del total de los ministros.

A continuación, se presentan los resultados del modelo logit con efectos marginales condicionales. Los resultados presentados apoyan parcialmente las hipótesis. Los ministros políticos tienen más posibilidades que sus pares de ser precandidatos. No obstante, el tiempo en el cargo no resulta significativo. Se comprueba la segunda hipótesis que asume que con independencia de la orientación ideológica la popularidad puede ser la variable que explique que un ministro sea considerado como precandidato.

Lo modelos de la tabla 3 están agrupados en dos. En el primero se presentan solo características personales. En este modelo se observa que el sexo y el capital familiar son variables que tienen significación estadística. El resto de los atributos personales tienen impacto en la posibilidad de que el ministro sea precandidato. La edad es negativa, es decir, en la medida que aumenta la posibilidad de ser precandidato disminuye. Tienen un impacto positivo los elementos específicos del perfil. La experiencia previa a 1973 tiene impacto. Los perfiles construidos en la tipología son significativos. Los ministros políticos, technopols y tecnócratas tienen treinta puntos más de posibilidades de ser candidatos, en comparación con los outsiders. En el segundo modelo se incorporan variables que no están relacionadas con las características del sujeto. Al agregar existen variaciones interesantes. Aunque muy bajo, ahora el sexo pasa a ser significativo. Es decir, ser hombre aumenta en 1,86 puntos las posibilidades de ser precandidato. La edad mantiene el mismo signo, se confirma que en la medida que aumenta la posibilidad de ser precandidato disminuye. También se confirma que los ministros políticos, technopols y tecnócratas tienen más posibilidades de ser precandidatos que los outsiders. Lo mismo ocurre con la experiencia previa a 1973. De las nuevas variables las únicas que son significativas son la popularidad y la orientación ideológica. En la medida que los partidos son más de derecha tienen más posibilidades de que sus militantes y adherentes que están en el gabinete sean precandidatos. Del mismo modo, los ministros que son incluidos en los listados de la encuesta del Centro de Estudios Públicos tienen más chances que no. 
Tabla 3. Logit precandidatos en el gabinete 1990-2014.

\begin{tabular}{|c|c|c|c|c|}
\hline & \multicolumn{2}{|c|}{ Modelo 1} & \multicolumn{2}{|c|}{ Modelo 2} \\
\hline & Coeficientes & $\begin{array}{c}\text { Efectos } \\
\text { marginales }\end{array}$ & Coeficientes & $\begin{array}{c}\text { Efectos } \\
\text { marginales }\end{array}$ \\
\hline Sexo & $\begin{array}{c}0.455 \\
(0.277)\end{array}$ & 0.0107 & $\begin{array}{l}1.843^{*} \\
(1.850)\end{array}$ & 0.0186 \\
\hline Edad & $\begin{array}{l}-0.383^{* * *} \\
(-2.109)\end{array}$ & -0.00901 & $\begin{array}{l}-1.033^{* * * *} \\
(-3.606)\end{array}$ & -0.0104 \\
\hline Políticos & $\begin{array}{c}14.36^{\text {***** }} \\
(10.95)\end{array}$ & 0.338 & $\begin{array}{c}10.78^{* * * *} \\
(8.074)\end{array}$ & 0.109 \\
\hline Technopols & $\begin{array}{l}14.00 * * * \\
(12.77)\end{array}$ & 0.329 & $\begin{array}{l}11.42^{\text {**** }} \\
(5.666)\end{array}$ & 0.115 \\
\hline Tecnócratas & $\begin{array}{l}16.40^{* * * *} \\
(15.99)\end{array}$ & 0.386 & $\begin{array}{l}13.26^{* * * *} \\
(12.08)\end{array}$ & 0.134 \\
\hline Capital familiar & $\begin{array}{l}-0.0446 \\
(-0.0479)\end{array}$ & -0.00105 & $\begin{array}{l}-0.543 \\
(-0.706)\end{array}$ & -0.00548 \\
\hline $\begin{array}{l}\text { Experiencia Pre } \\
73\end{array}$ & $\begin{array}{l}1.434 \% * \\
(2.519)\end{array}$ & 0.0337 & $\begin{array}{l}2.756^{* * * *} \\
(4.729)\end{array}$ & 0.0278 \\
\hline Días en el cargo & & & $\begin{array}{c}0.000576 \\
(1.035)\end{array}$ & $5.81 \mathrm{e}-06$ \\
\hline Variación PIB & & & $\begin{array}{l}-0.0438 \\
(-0.792)\end{array}$ & -0.000442 \\
\hline Variación IPC & & & $\begin{array}{l}-0.0870 \\
(-0.753)\end{array}$ & -0.000877 \\
\hline Popularidad & & & $\begin{array}{l}4.121^{* * * *} \\
(4.815)\end{array}$ & 0.0416 \\
\hline $\begin{array}{l}\text { Ministerios } \\
\text { sociales }\end{array}$ & & & $\begin{array}{c}0.797 \\
(0.499)\end{array}$ & 0.00803 \\
\hline $\begin{array}{l}\text { Ministerios } \\
\text { políticos }\end{array}$ & & & $\begin{array}{c}0.0735 \\
(0.0891)\end{array}$ & 0.000741 \\
\hline $\begin{array}{l}\text { Ministerios } \\
\text { económicos }\end{array}$ & & & $\begin{array}{c}1.117 \\
(0.590)\end{array}$ & 0.0113 \\
\hline $\begin{array}{l}\text { Orientación } \\
\text { ideológica del } \\
\text { partido }\end{array}$ & & & $\begin{array}{l}0.308 \% * \\
(2.031)\end{array}$ & 0.00311 \\
\hline $\begin{array}{l}\text { Militar en Partido } \\
\text { presidente }\end{array}$ & & & $\begin{array}{c}-0.333 \\
(-0.606)\end{array}$ & -0.00336 \\
\hline Constant & \multicolumn{2}{|c|}{$-17.51^{* * * * * *}(-5.490)$} & \multicolumn{2}{|c|}{$-18.16^{* * * *}(-8.779)$} \\
\hline Pseudo R2 & \multicolumn{2}{|c|}{0.0922} & \multicolumn{2}{|c|}{0.4789} \\
\hline $\begin{array}{l}\text { Log pseudolikeli- } \\
\text { hood }\end{array}$ & \multicolumn{2}{|c|}{-48.000898} & \multicolumn{2}{|c|}{-25.06602} \\
\hline Observations & \multicolumn{2}{|c|}{232} & \multicolumn{2}{|c|}{200} \\
\hline
\end{tabular}

Robust z-statistics in parentheses ${ }^{* * * *} \mathrm{p}<0.01,{ }^{* * *} \mathrm{p}<0.05,{ }^{*} \mathrm{p}<0.1$ 
Un elemento que habría que considerar es que el tipo de ministerio no tiene incidencia en las posibilidades de ser considerado como precandidato presidencial. Contrario a lo que se podría esperar, los ministerios que tienen más contacto con la ciudadanía no tienen efectos en la variable dependiente. Esto en gran parte se puede explicar porque los ministros que fueron considerados como precandidatos estaban distribuidos en todos los tipos de ministerios. Incluso, en aquellos más técnicos como Obras Públicas. Del mismo modo y también contrario a lo esperado, para un ministro, servir en un ministerio político no aumenta las posibilidades de precandidatura presidencial al igual que militar en el partido del Presidente. Esto rechaza algunos de los supuestos de la literatura especializada, que sugieren que el partido del Presidente tiene alguna ventaja sobre sus socios. Por último, el trabajo testeó el capital familiar como una variable que podría ser importante para la configuración de precandidaturas. Al igual que trabajos anteriores no resulta ser una variable que dé ventajas. Ya sea para ser precandidato, para ser consejero regiona ${ }^{67}$ o para sobrevivir en el cargo ${ }^{68}$.

\section{Conclusiones}

El análisis de los gabinetes es relevante para la ciencia política, ya que se realiza sobre una institución en la cual se articulan y diseñan políticas; se vincula el sistema de gobierno con los partidos y se demuestra la capacidad del Presidente para ejercer su liderazgo. En el ejercicio del liderazgo es que el Presidente podría querer tener en su equipo de trabajo más cercano (el gabinete) a un potencial candidato para sucederlo. Por lo tanto, estudiar a los precandidatos y candidatos que han pasado por el gabinete puede entregar elementos importantes sobre el funcionamiento del sistema político. Se ha estudiado la formación y mantención de los gabinetes, el estudio de las carreras, capacidades y redes de los ministros, los motivos que explican la llegada de una persona al gabinete la importancia relativa de algunos, así como su aporte en procesos políticos. No obstante, carreras posteriores una vez fuera del cargo y, por lo tanto, el impacto de los gabinetes en los sujetos es algo reciente ${ }^{69}$.

Aunque el caso chileno permite identificar características de los ministros que pueden ser candidatos, aun es necesario avanzar hacia la teorización y sistematización de experiencias en otros presidencialismos. El trabajo permitió iniciar debates sobre los alcances que tendría generar trabajos de este tipo para otros casos de la región, ya que investigaciones sobre estas temáticas pueden entregar importantes evidencias sobre cómo se distribuye el poder, cuáles son sus valoraciones sobre el

67. GONZÁLEZ-BUSTAMANTE (2014).

68. OLIVARES (2017).

69. GONZÁLEZ-BUSTAMANTE y GARRIDO (2018). 
sistema político y sobre la centralidad de los partidos políticos en la formación de gabinetes. Desde un punto de vista práctico se generó conocimiento relevante, al confirmar a un grupo de variables como las que más influyen en la posibilidad de que alguien del gabinete sea precandidato en Chile. Se demuestra que ser un ministro que es popular, independiente de la cartera donde sirva, tienen posibilidades de ser precandidato y después candidato.

\section{Referencias bibliográficas}

ALEMÁN, Eduardo y TSEBELIS, George (2012). "Partidos políticos y coaliciones de gobierno en las Américas". Política, Revista de Ciencia Política 50(2), 5-32.

ALTMAN, David (200o). "The Politics of Coalition Formation and Survival in Multiparty Presidential Democracies: The Case of Uruguay, 1989-1999". Party Politics, 6(3), 259-283

ALTMAN, David y CASTIGLIONNI, Roxana (2008).“Cabinet Determinants of Structural Reforms in Latin America, 1985-200o". The Developing Economies, 46(1), 1-25.

ALTMAN, David (2008). "Political Recruitment and Candidate Selection in Chile (1990-2003). The Executive Branch". En SIAVELIS, Peter y MORGENSTERN Scott (eds.), Pathways to Power: Political Recruitment and Candidate Selection in Latin America (pp. 241-270). University Park, Pa: Penn State University Press.

AMORIM NETO, Octavio (1998). Cabinet Formation in Presidential Regimes: An Analysis of 10 Latin American Countries. Presentado en Meeting of the Latin American Studies Association, Illinois.

ANGELL, Allan (2005). Elecciones presidenciales, democracia y partidos políticos en el Chile post Pinochet. Santiago: Centro de Estudios Bicentenario.

ARELLANO, Juan Carlos, TORO, Sergio y NOVOA, Romina (2018). ¿Cómo cambian los gabinetes? Las condiciones históricas de la sobrevivencia ministerial en el presidencialismo chileno. Revista Chilena de Derecho y Ciencia Política, 9, 114-146.

AUTH, Pepe (2005). Las primarias en la Concertación. Un camino sin retorno. Agenda Pública, IV (7), 10-15.

AVENDAÑO, Octavio (2010). Influencia partidaria y distribución de los gabinetes en Chile 1994-2010. Ponencia presentada en el Vo Congreso Latinoamericano de Ciencia Política ALACIP, Buenos Aires.

AVENDAÑO, Octavio (2010b). "El giro hacia la derecha en las últimas elecciones chilenas". Revista de Sociología, 24, 167-178.

AVENDAÑO, Octavio y DÁVILA, Mireya (2012). "Rotación ministerial y estabilidad coalicional en Chile, 1990-2010”. Política. Revista de Ciencia Política, 50(2), 87108. 
BLONDEL, Jean (1985). Government Ministers in the Contemporary World. London: SAGE Publications.

BOENINGER, Edgardo (1997). Democracia en Chile. Lecciones para la gobernabilidad. Santiago: Andrés Bello.

DÁVILA, Mireya (2011). Governing Together: The Concertación Administrations in Chile (1990-2009). (Tesis de Doctorado). University of North Carolina at Chapel Hill, Estados Unidos.

DÁVILA, Mireya y AVENDAÑO, Octavio (2018). “Together we govern portfolio allocation in Chile (1990-2014)”. En CAMERLO Marcelo y MARTÍNEZGALLARDO, Cecilia (eds.), Government formation and minister turnover in presidential cabinets: comparative analysis in the Americas. London and New York: Routledge.

DÁVILA, Mireya, OLIVARES, Alejandro y AVENDAÑO, Octavio (2013). "Los gabinetes de la Concertación en Chile (1990-2010)". América Latina Hoy, 64, 67-94.

DELAMAZA, Gonzalo (2011). "Elitismo democrático, líderes civiles y tecnopolítica en la reconfiguración de las élites políticas”. En JOIGNANT, Alfredo y GÜELL, Pedro (Eds.), Notables, tecnócratas y mandarines: elementos de sociología de las elites en Chile, 1990-2010. Santiago: Ediciones Universidad Diego Portales.

DOGAN, Mattei (1979). "How to Become a Cabinet Minister in France: Career Pathways, 1870-1978". Comparative Politics, 12(1), 1-25.

EGANA, Rodrigo y CHATEAU, Jorge (2011). "El Centro de Gobierno: Lecciones de la experiencia chilena durante los gobiernos de la Concertación (1990-2010)". Estado, Gobierno y Gestión Pública, $\mathrm{N}^{\circ}$ 17, 137-191.

FUENTES, Claudio (1999). "Partidos y coaliciones en el Chile de los 9o. Entre pactos y proyectos”. En DRAKE Paul e JAKSIC, Iván (comp.) El modelo chileno. Democracia y desarrollo en los noventa. Santiago: LOM.

FUNK, Robert (2006). El gobierno de Ricardo Lagos: la nueva vía chilena hacia el socialismo. Santiago: Ediciones Universidad Diego Portales.

GARRETÓN, Manuel Antonio (2003). "La (in)conducción política del segundo gobierno democrático”. En Muñoz, Oscar y ESTEFONI, Carolina (Eds.), El período del Presidente Frei Ruiz-Tagle: reflexiones sobre el segundo gobierno concertacionista. Santiago: Editorial Universitaria.

GARRIDO, Carolina y NAVIA, Patricio (2005). "Candidatos fuertes en la Concertación: ¿Seguro para subcampeones o prevalencia de los dos tercios?”. Estudios Públicos, (99), 165-194.

GONZÁLEZ-BUSTAMANTE, Bastián (2013). "Factores de acceso y permanencia de la élite política gubernamental en Chile (1990-2010)”. Política. Revista de Ciencia Política, 51(1), 119-153. 
GONZÁLEZ-BUSTAMANTE, Bastián (2014). “Elección directa de consejeros regionales 2013. Rendimiento del capital político, familiar y económico en una nueva arena electoral en Chile". Política. Revista de Ciencia Política, 52(2), 49-91.

GONZÁLEZ-BUSTAMANTE, Bastián y GARRIDO, Luis (2018). “Socialización, trayectorias y poscarrera de ministros en Chile, 1990-2010". Política y gobierno, 25(1), 31-64.

GONZÁLEZ-BUSTAMANTE, Bastián y OLIVARES, Alejandro (2015). "Rotación de subsecretarios en Chile: una exploración de la segunda línea gubernamental (1990-2014)". Revista de Gestión Pública, 4(2), 150-191.

GONZÁLEZ-BUSTAMANTE, Bastián y OLIVARES, Alejandro (2016). "Cambios de gabinete y supervivencia de los ministros en Chile durante los gobiernos de la Concertación (1990-2010)". Colombia Internacional, 87, 81-108.

GONZÁLEZ-BUSTAMANTE, Bastián y OLIVARES, Alejandro (2018). "La élite política gubernamental en Chile: Supervivencia de ministros (1990-2014)". En A. CoDATO y F. ESPINOZA (Eds.), Élites en las Américas: diferentes perspectivas. Brasil y Argentina: Editora UFPR y Ediciones UNGS.

HAZAN, Reuven, y RAHAT, Gideon (2010). Democracy within Parties: Candidate Selection Methods and Their Political Consequences. Oxford University Press.

HIDALGO, Paulo (2011). El ciclo político de la Concertación, 199o-2010. Santiago: Uqbar Editores.

HOSMER, David, LEMESHOW, Stanley y STURDIVANT, Rodney (2013). Applied logistic regression. Hoboken, New Jersey: Wiley.

JOIGNANT, Alfredo (2014). "El capital político familiar: Ventajas de parentela y concentraciones de mercado en las elecciones generales chilenas de 2013". Política. Revista de Ciencia Política, 52(2), 13-48.

JOIGNANT, Alfredo (2011). "The Politics of Technopols: Resources, Political Competence and Collective Leadership in Chile, 1990-2010". Journal of Latin American Studies, 43(3), 517-546.

LUNA, Juan Pablo y SELIGSON, Mitchell (2007). Cultura política de la democracia en Chile: 2006. Santiago: LAPOP y Universidad Católica.

MAILLET, A., GONZÁLEZ-BUSTAMANTE, B., y OLIVARES, A (2016). ¿Puerta giratoria? Análisis de la circulación público-privada en Chile (200o-2014). Chile: PNUD.

MARTÍNEZ-GALLARDO, Cecilia (2012a). "Cabinet Stability and Policymaking in Latin America”. En J. SANTISO y J. DAYTON-JOHNSON (Eds.), The Oxford Handbook of Latin American Political Economy. Oxford: Oxford University Press. 
MARTÍNEZ-GALLARDO, Cecilia (2012b). "Out of the Cabinet What Drives Defections From the Government in Presidential Systems?". Comparative Political Studies, 45(1), 62-90.

MORALES, Mauricio y NAVIA Patricio (2010). El sismo electoral de 20o9. Cambio y continuidad en las preferencias politicas de los chilenos. Santiago: Ediciones Universidad Diego Portales.

NAVARRETE, Bernardo (2005). "Las primarias como mecanismo de candidatos. La experiencia de 1993". Agenda Pública, Año IV, No 7, 20-26.

NAVIA, Patricio (2005). "La elección presidencial de 1993. Una elección sin incertidumbre”. En SAN FRANCISCO, Alejandro y SOTO, Ángel (Eds.), Camino a La moneda: las elecciones presidenciales en la historia de Chile 1920-20oo. Santiago: Pontificia Universidad Católica, Instituto de Historia y Centro de Estudios Bicentenario.

NAVIA, Patricio y JOIGNANT, Alfredo (200o). "Las Elecciones Presidenciales de 1999: La Participación Electoral y el Nuevo Votante Chileno”. En FLACSO (Ed.), Nuevo gobierno: desafíos de la reconciliación, Chile, 1999-20oo. Santiago: FLACSO-Chile.

OLIVARES, Alejandro (2017). La conformación de gabinetes y supervivencia de ministros en Chile y Uruguay durante los períodos pre y post golpes de Estado (Tesis de Doctorado). Universidad de Chile, Santiago.

OLIVARES, Alejandro, DÁVILA, Mireya y BAEZA, Jaime (2015). "Los gabinetes ministeriales en la democracia chilena post-1990: Un caso de estabilidad, continuidad histórica y negociación inter-partidaria". Revista DAAPGE, 24(15), 7-31.

OTANO, Rafael (1995). Crónica de la transición. Santiago: Editorial Planeta. OTANO, Rafael (2006). Nueva crónica de la transición. Santiago, Chile: Lom Ediciones.

REHREN, Alfredo (1993). "Liderazgo presidencial y democratización en el cono sur de América Latina”. Revista de ciencia política, 14(1-2), 63-87.

RODRÍGUEZ, Juan (2011). Los ministros de la España democrática. Madrid: Centro de Estudios Constitucionales.

RUBIO, Pablo (2014). Los civiles de Pinochet: la derecha en el régimen militar chileno, 1983-1990. Santiago: Ediciones de la Dirección de Bibliotecas, Archivos y Museos, Centro de Investigaciones Diego Barros Arana, colección Sociedad y Cultura.

SIAVELIS, Peter y GALVÁN, Humberto (2015). "Chile. Ministerial selection and deselection". En K. DOWDING y P. DUMONT (Eds.). The Selection of Ministers around the World (pp. 244-263). Londres: Routledge. 
SIAVELIS, Peter y MORGENSTERN, Scott (2009). "Reclutamiento y selección de candidatos en América Latina: un marco para el análisis". En FREIDENBERG, F. y ALCÁNTARA M. (coordinadores) Selección de candidatos, política partidista y rendimiento democrático. México. IUI-UNAM-TEDF-UAM.

SIAVELIS, Peter (1999). "Continuidad y transformación del sistema de partidos en una transición "modelo". En, Paul DRAKE e Iván JAKSIC (compiladores) El modelo chileno. Democracia y desarrollo en los noventa. Santiago: LOM.

SILVA, Patricio (2010). En el nombre de la razón: tecnócratas y política en Chile. Santiago: Ediciones Universidad Diego Portales.

TRICOT, Tokichen (2012). "Movimiento de estudiantes en Chile: Repertorios de acción colectiva ¿algo nuevo?”. Revista F@ro, (15). Disponible en www. revistafaro.cl/index.php/Faro/article/view/63 [consultado el 25 de marzo de 2018].

VARAS, Augusto (2010). "Del éxito al fracaso concertacionista. El gobierno de Michelle Bachelet y la derrota electoral de 2010". En QUIROGA, Yerko y ENSIGNIA, Jaime (Eds.), Chile en la Concertación (1990-2010) Una mirada crítica, balance y perspectivas Tomo II. Santiago: FES.

VON BÜLOW, Marisa y BIDEGAINPONTE, Germán (2015). "It Takes Two to Tango: Students, Political Parties, and Protest in Chile (2005-2013)". En ALMEIDA, Paulo y CORDERO ULATE, Allen (Eds.), Handbook of Social Movements across Latin America (pp. 179-194). Dordrecht: Springer Netherlands.REVISTA CHILENA DE DERECHO Y CIENCIA POLÍTICA 